ACCEPTED By ApJL

Preprint typeset using $\mathrm{LATEX}_{\mathrm{E}}$ style emulateapj v. 5/2/11

\title{
THE LYMAN ALPHA REFERENCE SAMPLE: EXTENDED LYMAN ALPHA HALOS PRODUCED AT LOW
} DUST CONTENT

\author{
Matthew Hayes ${ }^{2,3}$, Göran Östlin ${ }^{4}$, Daniel Schaerer ${ }^{5,3}$, Anne Verhamme ${ }^{5}$, J. Miguel Mas-Hesse ${ }^{6}$, Angela Adamo ${ }^{7}$, \\ Hakim AteK $^{8}$, John M. Cannon ${ }^{9}$, Florent Duval ${ }^{4}$, Lucia Guaita ${ }^{4}$, E. Christian Herenz ${ }^{10}$, Daniel Kunth ${ }^{11}$, Peter \\ Laursen $^{12}$, Jens Melinder ${ }^{4}$, Ivana Orlitova ${ }^{5,13}$, Héctor Otí-Floranes ${ }^{14,15,6}$, And Andreas SandBerg ${ }^{4}$ \\ Accepted by ApJL
}

\begin{abstract}
We report on new imaging observations of the Lyman alpha emission line (Ly $\alpha$ ), performed with the Hubble Space Telescope, that comprise the backbone of the Lyman alpha Reference Sample (LARS). We present images of 14 starburst galaxies at redshifts $0.028<z<0.18$ in continuum-subtracted $\operatorname{Ly} \alpha, \mathrm{H} \alpha$, and the far ultraviolet continuum. We show that $\mathrm{Ly} \alpha$ is emitted on scales that systematically exceed those of the massive stellar population and recombination nebulae: as measured by the Petrosian 20 percent radius, $R_{\mathrm{P} 20}$, Ly $\alpha$ radii are larger than those of $\mathrm{H} \alpha$ by factors ranging from 1 to 3.6, with an average of 2.4. The average ratio of Ly $\alpha$-to-FUV radii is 2.9. This suggests that much of the Ly $\alpha$ light is pushed to large radii by resonance scattering. Defining the Relative Petrosian Extension of Ly $\alpha$ compared to $\mathrm{H} \alpha, \xi_{\mathrm{Ly} \alpha}=R_{\mathrm{P} 20}^{\mathrm{Ly} \alpha} / R_{\mathrm{P} 20}^{\mathrm{H} \alpha}$, we find $\xi_{\mathrm{Ly} \alpha}$ to be uncorrelated with total Ly $\alpha$ luminosity. However $\xi_{\mathrm{Ly} \alpha}$ is strongly correlated with quantities that scale with dust content, in the sense that a low dust abundance is a necessary requirement (although not the only one) in order to spread Ly $\alpha$ photons throughout the interstellar medium and drive a large extended Ly $\alpha$ halo.

Subject headings: Physical data and processes: Radiative transfer — Galaxies: evolution — Galaxies: formation - Galaxies: starburst - Cosmology: observations
\end{abstract}

\section{INTRODUCTION}

The Lyman alpha emission line ( $\operatorname{Ly} \alpha)$, emitted by the spontaneous de-excitation over the $n=2 \rightarrow 1$ electronic transition in neutral hydrogen (HI), is now an

matthew.hayes@irap.omp.eu

1 Based on observations made with the NASA/ESA Hubble Space Telescope, obtained at the Space Telescope Science Institute, which is operated by the Association of Universities for Research in Astronomy, Inc., under NASA contract NAS 5-26555. These observations are associated with program \#12310.

${ }^{2}$ Université de Toulouse; UPS-OMP; IRAP; Toulouse, France

${ }^{3}$ CNRS; IRAP; 14, avenue Edouard Belin, F-31400 Toulouse, France

${ }^{4}$ Department of Astronomy, Oskar Klein Centre, Stockholm University, AlbaNova University Centre, SE-106 91 Stockholm, Sweden

${ }^{5}$ Geneva Observatory, University of Geneva, 51 Chemin des Maillettes, CH-1290 Versoix, Switzerland

6 Centro de Astrobiología (CSIC-INTA), Departamento de Astrofísica, POB 78, 28691 Villanueva de la Cañada, Spain.

${ }^{7}$ Max Planck Institute for Astronomy, Königstuhl 17, D-69117 Heidelberg, Germany.

${ }^{8}$ Laboratoire dAstrophysique, École Polytechnique Fédérale de Lausanne (EPFL), Observatoire, CH-1290 Sauverny, Switzerland.

${ }^{9}$ Department of Physics and Astronomy, Macalester College, 1600 Grand Avenue, Saint Paul, MN 55105, USA.

10 Leibniz-Institut für Astrophysik (AIP), An der Sternwarte 16, D-14482 Potsdam, Germany.

${ }^{11}$ Institut d'Astrophysique de Paris, UMR 7095 CNRS \& UPMC, 98 bis Bd Arago, 75014 Paris, France.

12 Dark Cosmology Centre, Niels Bohr Institute, University of Copenhagen, Juliane Maries Vej 30, 2100 Copenhagen, Denmark.

13 Astronomical Institute of the Academy of Sciences, Bočnı' II 1401/1a, CZ-141 31 Praha 4, Czech Republic.

14 Instituto de Astronomía, Universidad Nacional Autónoma de México, Apdo. Postal 106, Ensenada B. C. 22800 Mexico

15 Dpto. de Física Moderna, Facultad de Ciencias, Universidad de Cantabria, 39005 Santander, Spain established observational probe of evolving galaxies in the high- $z$ Universe (Cowie \& Hu 1998; Rhoads et al. 2000). Exploitation of Ly $\alpha$ has resulted in significant galaxy surveys (Ouchi et al. 2008; Nilsson et al. 2009; Guaita et al. 2010; Adams et al. 2011), the next generations of which will recover vast numbers of galaxies. However the HI abundance in most galaxies, combined with the large Ly $\alpha$ absorption cross section of groundstate hydrogen, suggests that most Ly $\alpha$ will be absorbed and re-scattered by the same transition that created it. Thus most $\operatorname{Ly} \alpha$ photons are thought to be subject to multiple scattering events as they encounter neutral gas, resulting in a complicated radiative transport Neufeld 1990; Verhamme et al. 2006; Laursen et al. 2009).

Because $\mathrm{HI}$ is often found at distances that exceed the size of stellar disks and star-forming regions (Yun et al. 1994; Meurer et al. 1996; Cannon et al. 2004), characteristic Ly $\alpha$ scale lengths may be expected to be substantially larger than those of, for example, the FUV continuum or $\mathrm{H} \alpha$. Indeed this has been well observed at high $z$ (e.g. Fynbo et al. 2001; Rauch et al. 2008; Steidel et al. 2011, although see also Feldmeier et al. 2013) and low (Mas-Hesse et al. 2003; Ostlin et al. 2009), and studied extensively by simulation (Laursen et al. 2009; Barnes \& Haehnelt 2010; Zheng et al. 2011; Verhamme et al. 2012).

In this Letter we present images from the Lyman alpha Reference Sample (LARS). The LARS program (Östlin et al., in prep; Hayes et al., in prep) is targeting $14 \mathrm{UV}$ selected star-forming galaxies in the nearby Universe, all of which have been imaged in $\mathrm{Ly} \alpha, \mathrm{H} \alpha, \mathrm{H} \beta$, and five UV/optical continuum bands. Many other observations, both in hand and ongoing, are providing gas covering fractions and kinematics, and measuring the HI mass and extent directly. HST imaging allows us to probe spatial 

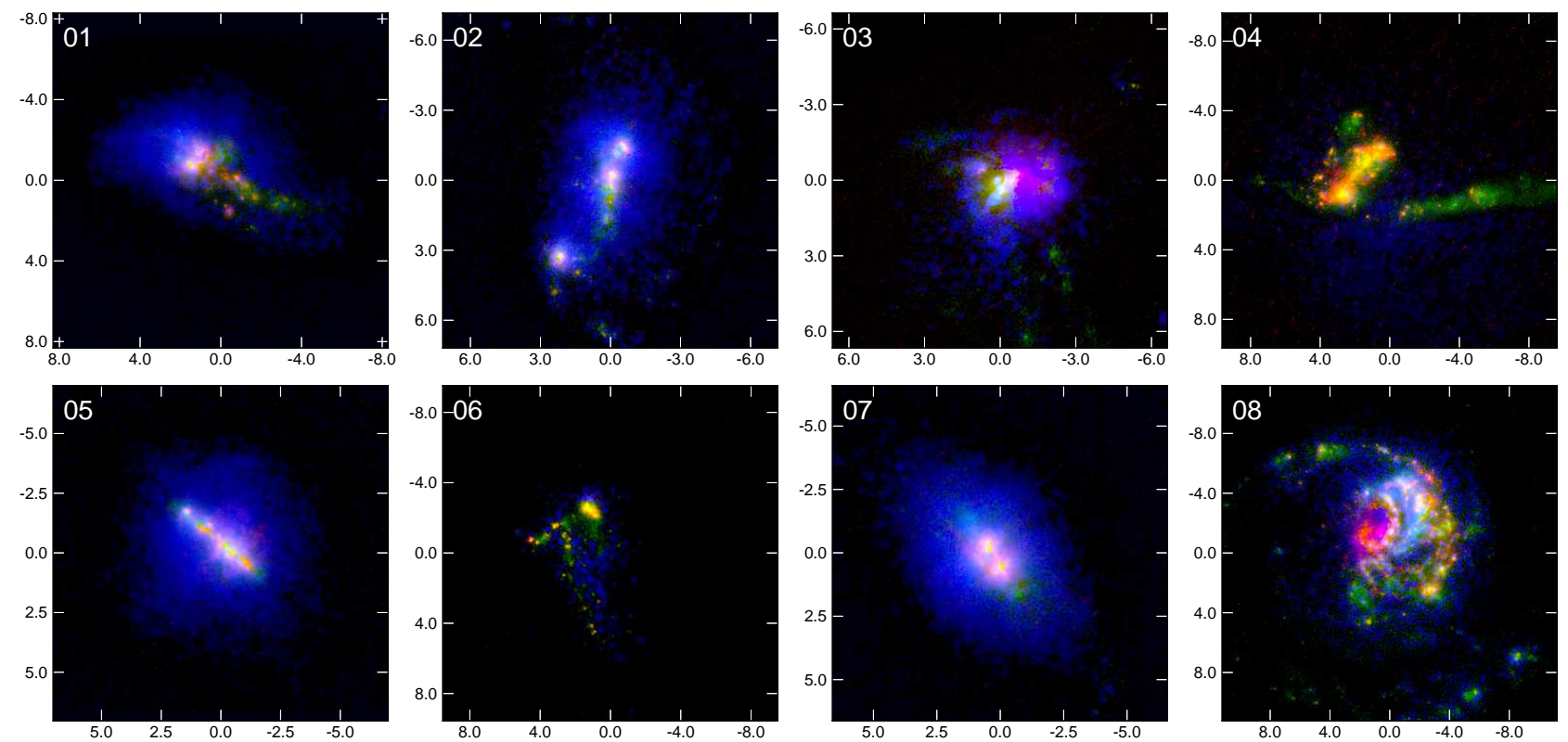

Figure 1. False-color images of the LARS galaxies 01 to 08 . Red encodes continuum-subtracted H $\alpha$, green the FUV continuum, and blue shows continuum-subtracted Ly $\alpha$. Images have been adaptively filtered to show detail. Scales in kpc are given on the side. Intensity scales are logarithmic, with intensity cut levels set to show detail.

scales down to $28 \mathrm{pc}$ in individual galaxies, quantify the extent of Ly $\alpha$, and compare it with other wavelengths and derived properties. This Letter discusses the extension of Ly $\alpha$ radiation. In Section 2 we briefly summarize the data and show the new images. In Section 3 we quantify the sizes of the galaxies in Ly $\alpha, \mathrm{FUV}$, and $\mathrm{H} \alpha$, and discuss them with reference to high- $z$ measurements in Section 4. In Section [5 we show how a low dust content seems to be a necessary prerequisite in order to produce this extended emission. We assume a cosmology of $\left(H_{0}, \Omega_{\mathrm{M}}, \Omega_{\Lambda}\right)=\left(70 \mathrm{~km} \mathrm{~s}^{-1} \mathrm{Mpc}^{-1}, 0.3,0.7\right)$.

\section{LARS IMAGES}

LARS consists of 14 star-forming galaxies selected by FUV luminosity from the GALEX all-sky surveys, and imaged with Hubble Space Telescope cameras ACS/SBC, ACS/WFC, and WFC3/UVIS. The sample selection, observations, and data processing are described in detail in Östlin et al. (in prep). FUV luminosities range between $\log \left(L_{\mathrm{FUV}} / L_{\odot}\right)=9.2$ and 10.7 , overlapping much of the luminosity range of Lyman-break Galaxy (LBG) surveys, and are listed in Table 1.

We use the Lyman alpha eXtraction software (LaXs, Haves et al. 2009) to produce continuum-subtracted Ly $\alpha$ and $\mathrm{H} \alpha$ images, corrected for underlying stellar absorption and contamination from [NII]. In 1 arcsec square boxes away from the targets we measure r.m.s. background noise of $5.7 \times 10^{-19} \mathrm{erg} \mathrm{s}^{-1} \mathrm{~cm}^{-2}$ in Ly $\alpha$, $2.1 \times 10^{-21} \mathrm{erg} \mathrm{s}^{-1} \mathrm{~cm}^{-2} \AA^{-1}$ in the FUV, and $6.8 \times$ $10^{-19} \mathrm{erg} \mathrm{s}^{-1} \mathrm{~cm}^{-2}$ in $\mathrm{H} \alpha$. Total Ly $\alpha$ luminosities range from 0 (non-detection) and $2 \times 10^{43} \mathrm{erg} \mathrm{s}^{-1}$ with a median of $8.1 \times 10^{41} \mathrm{erg} \mathrm{s}^{-1}$; roughly seven of the objects would be recovered by the deepest Ly $\alpha$ surveys (Hayes et al. in prep).

We present our first imaging results in this paper as a series of RGB composite images in Figures 1 and 2] In green we encode the far UV continuum, which traces the unobscured massive stars, and roughly incorporates the sites that produce the ionizing photons. In the red we show continuum-subtracted $\mathrm{H} \alpha$, which traces the nebulae where the aforementioned ionizing photons are reprocessed into the recombination line spectrum. The continuum subtracted Ly $\alpha$ observation is encoded in blue. The images have been adaptively smoothed using a variable Gaussian kernel (FILTER/ADAPTIVE in ESO/MIDAS), in order to enhance positive regions of low surface brightness emission. The intensity scaling of all the images is logarithmic, and the levels are set to show the maximum of structure and the level at which the faintest features fade into the background.

Immediately it can be seen that Ly $\alpha$ morphologies bear limited resemblance to those of the FUV and $\mathrm{H} \alpha$. In some cases Ly $\alpha$ appears to be almost completely absent: LARS 04 and 06 in particular show only small hints of Ly $\alpha$ emission that contribute negligibly towards filling in the global absorption, and the composites are dominated by UV and $\mathrm{H} \alpha$ light. Ly $\alpha$ is strongly absorbed, particularly in the central regions of these objects. Others show copious Ly $\alpha$ emission and reveal morphological structures that are not seen at other wavelengths. Most obviously, LARS 01, 02, 05, 07, 12, and 14 show largescale halos of Ly $\alpha$ emission that completely encompass the star-forming regions, although the same phenomenon is visible to some extent in all the objects, even the absorbers.

We have discussed this extended Ly $\alpha$ emission in depth in the past (Haves et al. 2005, 2007; Atek et al. 2008; Ostlin et al. 2009). However now, with an observational setup that is more sensitive to faint levels of Ly $\alpha$ and a larger and UV-selected sample (Östlin et al, in prep), we are able to robustly quantify and contrast these sizes and the relative extension of $\mathrm{Ly} \alpha$.

\section{APERTURES, SIZES AND GLOBAL QUANTITIES}

In order to quantify the sizes of the galaxies at various wavelengths, we adopt the Petrosian radius (Petrosian 

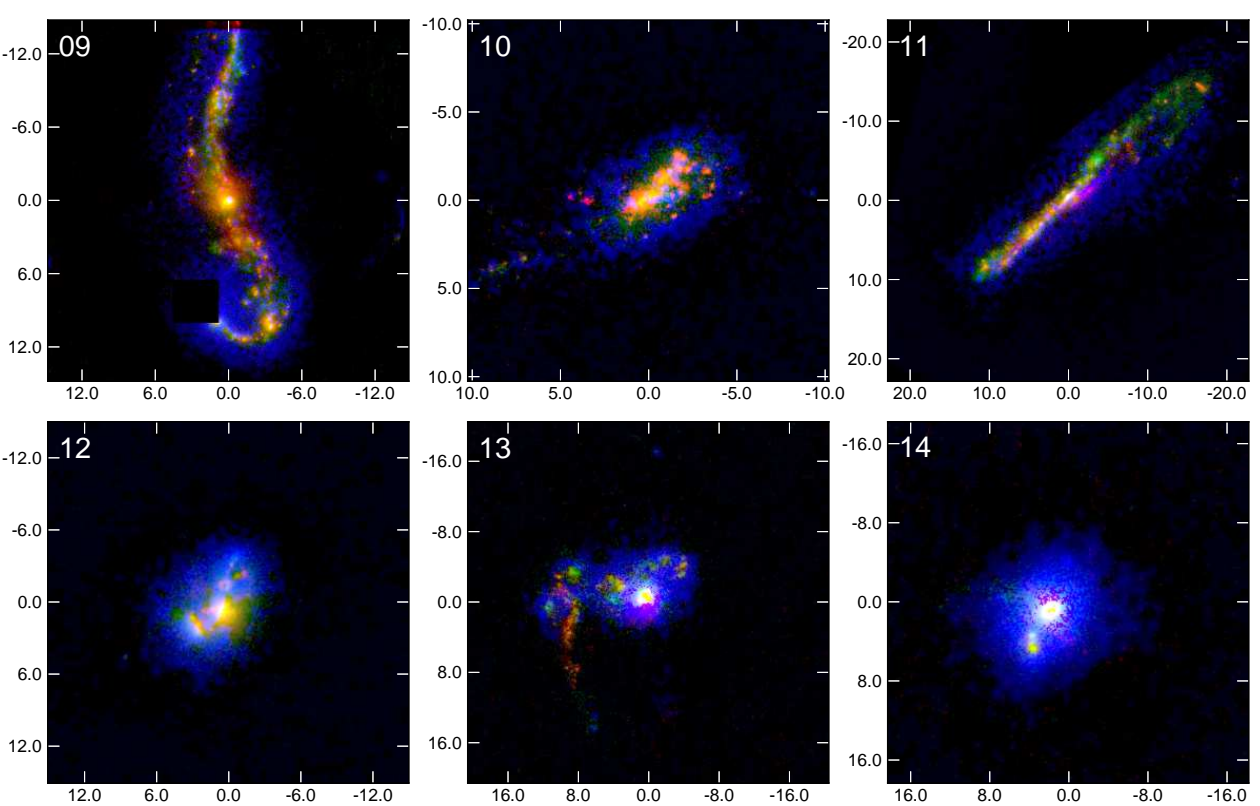

Figure 2. Same as Figure 1 except for LARS galaxies 09 to 14. The black square in LARS 09 masks a UV-bright field star.

1976) with index of $\eta=0.2$ : i.e. the radius, $R$, at which the local surface brightness is 20 percent the average surface brightness inside of $R$. In Hayes et al. (in prep) we will show the Ly $\alpha$ extent of some objects to be so large that ACS/SBC cannot capture the full flux, and hence measurements like 50 percent light radius are not robust. Indeed Petrosian radii were developed to be depthindependent measures of size. We note from experimentation, however, that very similar conclusions are reached using other definitions. The choice of $\eta=0.2$ gives a size for every Ly $\alpha$-emitting galaxy in the sample except LARS 09, for which even at the full extent of the SBC we do not come close to crossing the $\eta=0.2$ threshold. We reach the edge of the detector at $\eta \sim 1(R>12 \mathrm{kpc})$ and can expect the true extent of Ly $\alpha$ to be much larger. For the 11 galaxies in which $R_{\mathrm{P} 20}^{\mathrm{Ly} \alpha}$ is well measured, its determination is robust, and would not change were the observations deeper or the field-of-view larger. $R_{\mathrm{P} 20}$, is computed for $\mathrm{Ly} \alpha, \mathrm{H} \alpha$, and the FUV continuum, and listed in Table 1. Based upon aperture-matched $\mathrm{H} \alpha$ and $\mathrm{H} \beta$ imaging and standard Case $\mathrm{B}$ assumptions, we recover up to $60 \%$ of the intrinsic Ly $\alpha$ flux, although the median value is just $\sim 3 \%$ (Hayes et al. in prep).

We compare the light radii graphically in Figure 3. The plots show $R_{\mathrm{P} 20}^{\mathrm{Ly} \alpha}$ vs. $R_{\mathrm{P} 20}^{\mathrm{FUV}}$, a comparison that could be made at high- $z$, and $R_{\mathrm{P} 20}^{\mathrm{Ly} \alpha}$ vs. $R_{\mathrm{P} 20}^{\mathrm{H} \alpha}$, a comparison that more directly conveys the difference between the observed and intrinsic Ly $\alpha$ sizes. Clearly, though, there is little difference in the result: Ly $\alpha$ radii are, on average, substantially larger than corresponding FUV or $\mathrm{H} \alpha$ radii. In Table 1 we also report the Relative Petrosian Extension of Ly $\alpha$ compared to $\mathrm{H} \alpha, \xi_{\mathrm{Ly} \alpha}$, which is simply defined as $R_{\mathrm{P} 20}^{\mathrm{Ly} \alpha} / R_{\mathrm{P} 20}^{\mathrm{H} \alpha}$. 12 galaxies show net emission of $\operatorname{Ly} \alpha$, where all except for one (LARS 03) has $\xi_{\mathrm{Ly} \alpha}>1$. The galaxy with the largest extension is LARS 14, for which we measure $\xi_{\mathrm{Ly} \alpha}=3.6$. It is not clear whether globally absorbing galaxies LARS 04 and 06 become emitters on larger scales, but if so their $R_{\mathrm{P} 20}^{\mathrm{Ly} \alpha}$ must be larger than the radius of the SBC chip, implying that $\xi_{\text {Ly } \alpha}$ must exceed 5.3 and 13.4 , respectively. That would make them the most extended objects in the sample. Excluding these two galaxies, and also LARS 09 for which we can only provide a lower limit, the sample mean (median) is computed as 2.43 (2.28).

\section{RELEVANCE FOR HIGH-REDSHIFT STUDIES}

It is important to note that FUV radii imply that all the galaxies would be effectively unresolved by groundbased observations if they were at $z \gtrsim 2$. The largest is $8 \mathrm{kpc}$, which corresponds to the 1 arcsec resolution that could be expected from the seeing. However one of the objects has a Ly $\alpha$ radius of $15.5 \mathrm{kpc}$ : recovering this total flux at $z \sim 2$ would require an aperture of at least 2 arcsec. Some objects are also highly elongated and were they pushed to the high- $z$ Universe, much of their Ly $\alpha$ could also be unmeasured if circular apertures are used.

Ly $\alpha$ emission more extended than the FUV has been reported in numerous high- $z$ samples. Fynbo et al. (2003) remarked upon a few such objects at the brighter end of the luminosity distribution of the 27 narrowband selected galaxies, and the extremely deep spectroscopic observations of Rauch et al. (2008) uncovered 28 Ly $\alpha$ galaxies, ten of which were classified as extended. Samples of Ly $\alpha$ blobs (e.g. Matsuda et al. 2012; Prescott et al. 2012) may be many times the size of their counterpart galaxies, if indeed counterparts are identified at all. Here we report that every galaxy in the sample that emits Ly $\alpha$ does so by producing a halo; on average the halo is over twice the linear size of $\mathrm{H} \alpha$ and the FUV.

By stacking narrowband images of LBGs at $\langle z\rangle=$ 2.65, Steidel et al. (2011) reported Ly $\alpha$ halos that extend many tens of $\mathrm{kpc}$, probably probing the neutral circumgalactic medium (CGM) out to the virial radius. Subdividing the full sample by Ly $\alpha$ properties, the halos at radii larger than 20-30 physical kpc show very similar scale lengths in all subsamples (although different central surface brightnesses), even when central Ly $\alpha$ absorption is found. At small radii the subsamples exhibit 
Table 1

The LARS sample: properties and sizes.

\begin{tabular}{|c|c|c|c|c|c|c|c|c|c|c|c|c|}
\hline $\begin{array}{l}\text { LARS } \\
\text { ID } \\
(1)\end{array}$ & $\begin{array}{c}\text { Common name } \\
\text { (2) }\end{array}$ & $\begin{array}{l}\text { R.A. } \\
h: m: s \\
(3)\end{array}$ & $\begin{array}{c}\text { Dec. } \\
d: m: s \\
(4)\end{array}$ & $\begin{array}{c}z \\
(5)\end{array}$ & $\begin{array}{c}L_{\mathrm{FUV}} \\
L_{\odot} \\
(6)\end{array}$ & $\begin{array}{c}R_{\mathrm{P} 20}^{\mathrm{FUV}} \\
\mathrm{kpc} \\
(7)\end{array}$ & $\begin{array}{c}R_{\mathrm{P} 20}^{\mathrm{H} \alpha} \\
\mathrm{kpc} \\
(8)\end{array}$ & $\begin{array}{c}R_{\mathrm{P} 20}^{\mathrm{Ly} \alpha} \\
\mathrm{kpc} \\
(9)\end{array}$ & $\begin{array}{c}R_{\text {chip }}^{\mathrm{SBC}}(z) \\
\operatorname{kpc} \\
(10)\end{array}$ & $\begin{array}{c}\xi_{\mathrm{Ly} \alpha} \\
(11)\end{array}$ & $\begin{array}{c}\beta \text {-slope } \\
(12)\end{array}$ & $\begin{array}{c}\mathrm{H} \alpha / \mathrm{H} \beta \\
(13)\end{array}$ \\
\hline 01 & Mrk 259 & $13: 28: 44.0$ & $+43: 55: 49.9$ & 0.028 & 9.92 & 1.18 & 1.29 & 4.36 & 7.87 & 3.37 & -1.83 & 3.08 \\
\hline 03 & Arp 238 & $13: 15: 35.1$ & $+62: 07: 27.2$ & 0.031 & 9.52 & 0.84 & 0.97 & 0.75 & 8.68 & 0.77 & -0.57 & 5.18 \\
\hline 04 & & $13: 07: 28.2$ & $+54: 26: 50.7$ & 0.033 & 9.93 & 3.79 & 1.57 & $\ldots$ & 9.22 & $\ldots$ & -1.76 & 3.48 \\
\hline 05 & Mrk 1486 & $13: 59: 51.0$ & $+57: 26: 23.0$ & 0.034 & 10.0 & 0.93 & 1.24 & 3.24 & 9.49 & 2.61 & -2.09 & 3.06 \\
\hline 06 & KISSR 2019 & $15: 45: 44.5$ & $\begin{array}{l}+44: 15: 49.9 \\
\end{array}$ & 0.034 & 9.20 & 3.65 & 0.66 & & 9.48 & .01 & -1.85 & 2.96 \\
\hline 09 & IRAS $0820+2816$ & $08: 23: 54.9$ & $+28: 06: 22.8$ & 0.047 & 10.5 & 5.00 & 4.21 & $>12.0$ & 12.9 & $>2.85$ & -1.52 & 3.48 \\
\hline 10 & Mrk 0061 & $13: 01: 41.5$ & $+29: 22: 53.2$ & 0.057 & 9.74 & 2.34 & 2.63 & 5.49 & 15.5 & 2.08 & -1.36 & 3.93 \\
\hline 11 & $\ldots$ & $14: 03: 47.1$ & $+06: 28: 15.0$ & 0.084 & 10.7 & 8.00 & 6.81 & 15.5 & 22.1 & 2.27 & -1.50 & 4.60 \\
\hline 12 & SBS $0934+547$ & $09: 38: 13.5$ & $+54: 28: 25.3$ & 0.102 & 10.5 & 1.78 & 2.03 & 7.06 & 26.3 & 3.48 & -1.92 & 3.21 \\
\hline 13 & IRAS $0147+1254$ & $01: 50: 28.4$ & $+13: 08: 59.2$ & 0.147 & 10.6 & 3.83 & 4.68 & 8.12 & 36.0 & 1.74 & -1.53 & 4.07 \\
\hline 14 & $\ldots$ & $09: 26: 00.3$ & $+44: 27: 36.0$ & 0.181 & 10.7 & 0.79 & 1.62 & 5.86 & 42.7 & 3.62 & -2.22 & 3.13 \\
\hline
\end{tabular}

Note. - Coordinates (3 and 4) are J2000. Redshifts (5) are derived from SDSS. (6) are $\log \left(\nu L_{\nu}\right)$ in solar luminosities. (7-9) are the Petrosian radii with $\eta=0.2, R_{\mathrm{P} 20}$. (10) shows the physical scale corresponding to an angular size of 14 arcsec at the redshift of each galaxy - this corresponds to half the diametric size of the ACS/SBC (28 arcsec / 2) and describes the maximum usable scale to which we can probe Ly $\alpha$. (11) gives the relative extension of $\mathrm{Ly} \alpha$ relative toH $\alpha$. (12) gives the UV slope, $\beta$, derived from $\mathrm{HST}$ imaging. (13) gives the $\mathrm{H} \alpha / \mathrm{H} \beta$ ratio, derived from $\mathrm{SDSS}$ spectroscopy.
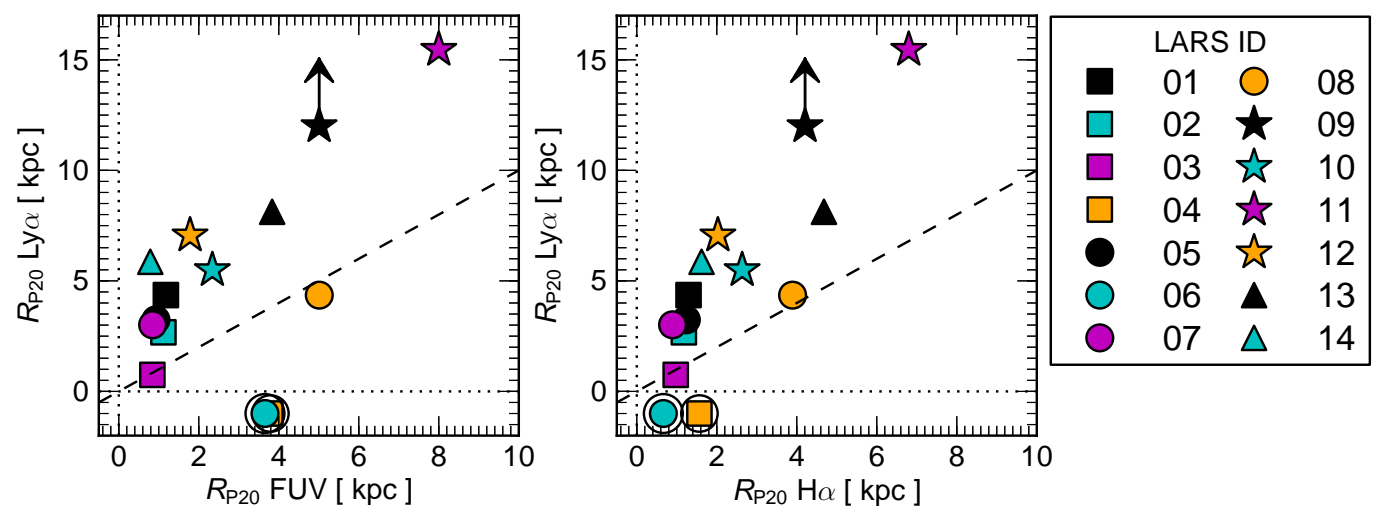

Figure 3. Comparison of the Petrosian radii $(\eta=0.2), R_{\mathrm{P} 20}$, in continuum-subtracted Ly $\alpha$, H $\alpha$, and the UV continuum. In cases where a galaxy is a net Ly $\alpha$ absorber its size is undefined, and $R_{\mathrm{P} 20}^{\mathrm{Ly} \alpha}$ has been set to a small negative value - it could in principle also be very large. The Left panel shows how Ly $\alpha$ sizes compare with the FUV, which can be similarly derived at high- $z$. The Right panel makes the same comparison against $R_{\mathrm{P} 20}^{\mathrm{H} \alpha}$, which directly contrasts the intrinsic and emitted Ly $\alpha$ sizes. When net Ly $\alpha$ emission is found it is systematically extended, taking mean values of $R_{\mathrm{P} 20}^{\mathrm{Ly} \alpha} / R_{\mathrm{P} 20}^{\mathrm{FUV}}=2.9$ (left) and $R_{\mathrm{P} 20}^{\mathrm{Ly} \alpha} / R_{\mathrm{P} 20}^{\mathrm{H} \alpha}=2.4$ (right).

profiles that differ markedly, dropping rapidly to $\sim 0$ for the Ly $\alpha$-absorbing sample but steepening by varying degrees in all others. Even the steepest central profiles, however, still run much flatter than those of the stellar continuum, and this change likely marks the onset of higher density gaseous disks or similar.

From the various $z \approx 2.7$ Ly $\alpha$ profiles of Steidel et al. (2011) we calculate $R_{\mathrm{P} 20}^{\mathrm{Ly} \alpha}$ using the same method as for our sample, and dividing by $R_{\mathrm{P} 20}^{\mathrm{FUV}}$ from the continuum profile we obtain $\xi_{\mathrm{Ly} \alpha}$ (now relative to the UV). These raw values range between $\xi_{\mathrm{Ly} \alpha}=3.8$ for the non-LAEs, and 5.9 for the LAE-only sample, and are notably bigger than our largest $\xi_{\mathrm{Ly} \alpha}$. However, under the assumption that the inner and outer profiles mark physically different regimes that may not be the same in low- $z$ galaxies, we also subtract the exponential halo fits of Steidel et al. (2011) and repeat the exercise; this yields a range of $\xi_{\mathrm{Ly} \alpha}=0.84$ to 2.0. This is now smaller than many of our values, although close to the average and the dispersion of the high- $z$ sample is obviously lost in the stacking process. On the other hand, the UV continuum profile of Steidel et al. (2011) is dominated by atmospheric seeing. If we instead use the continuum effective radius of $\mathrm{BM} / \mathrm{BX}$ galaxies and $\mathrm{LBG}$ from $\mathrm{HST}$ imaging (Mosleh et al. 2011) we compute $R_{\mathrm{P} 20}^{\mathrm{FUV}} \approx 5 \mathrm{kpc}$, which would increase all the $\xi_{\text {Ly } \alpha}$ quoted above by a factor of 2.5. $\xi_{\mathrm{Ly} \alpha}$ from the raw data would then become much larger than we measure in the local universe (up to $15)$, and $\xi_{\mathrm{Ly} \alpha}$ in halo-subtracted profiles that are roughly consistent (2.1 to 4.8$)$.

LARS observations probe scales far below the tens of kpc sampled at high- $z$ on a case-by-case basis. The galaxies likely include the range between, or roughly bracketing, the averaged subsamples of Steidel et al. (2011). Our imaging also suggests this extension to be a very common property of Ly $\alpha$-emitting galaxies, and its onset begins almost immediately in the inner few kpc: we find seven galaxies with FUV Petrosian radii below $2 \mathrm{kpc}$, five of which have corresponding Ly $\alpha$ radii three 

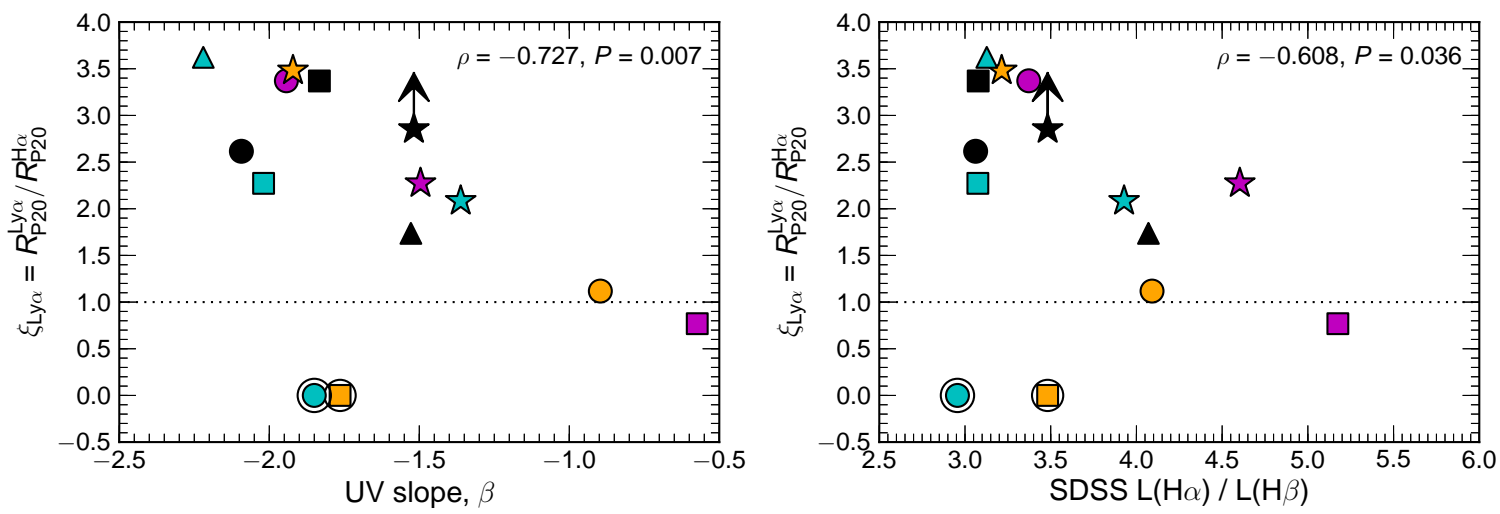

Figure 4. Correlations between the Ly $\alpha$ vs. $\mathrm{H} \alpha$ extension, $\xi_{\mathrm{Ly} \alpha}$, and measures of the galaxy reddening. The Left panel shows the UV slope, $\beta$ measured from HST imaging, while the Right panel shows the nebular attenuation measured from SDSS spectroscopy at H $\alpha$ and $\mathrm{H} \beta$. Net absorbing galaxies are set to zero and ringed, but could in principle also be very extended. Rank correlation coefficients of the Spearman $\rho$ test and the associated probability of the no-correlation hypothesis (accounting for ties and the small sample) are given in the top-right corners. Symbols are the same as Figure 3.

times larger.

It is also noteworthy that Steidel et al. (2011) find different median dust attenuations for the Ly $\alpha$-emitting and non-emitting subsamples, almost precisely as we did in Hayes et al. (2010). LAEs, which show extended central peaks, were determined to have stellar $E_{B-V}=0.09$ magnitudes (c.f. 0.085 in Hayes et al. 2010) while absorbers show $E_{B-V}=0.19$ (c.f. 0.23 for our $\mathrm{H} \alpha$-selected sample). Adopting the prescription of Meurer et al. (1999) the stellar $E_{B-V}$ measurements for the Steidel et al. samples correspond to $\beta$ slopes ${ }^{16}$ of -1.77 (LAEs) and -1.27 (Ly $\alpha$ absorbers). Bluntly accounting for a factor of 2.27 that connects stellar $E_{B-V}$ to its nebular equivalent in local starbursts (Calzetti et al. 2000), the same stellar $E_{B-V}$ would equate to $\mathrm{H} \alpha / \mathrm{H} \beta$ ratios of 3.5 (LAEs) and 4.4 (absorbers). In the next Section we will show case-by-case that Ly $\alpha$ halos systematically become more extended with decreasing dust contents.

\section{LYMAN ALPHA EXTENSION AND DUST CONTENTS}

In Hayes et al. (in prep) we compute many global properties for the sample, in order to study the processes complicit in $\operatorname{Ly} \alpha$ transport. Indeed that paper will include a complete analysis of correlations between $\operatorname{Ly} \alpha$ transmission, halo sizes, and many other properties; in this Letter we restrict ourselves to observables that scale with the dust content. It is noteworthy for the moment, however, that we find no correlation between $\xi_{\mathrm{Ly} \alpha}$ and the total Ly $\alpha$ luminosity. In Figure 4 we show how $\xi_{\text {Ly } \alpha}$ compares with both the UV continuum slope $\beta$ and the $\mathrm{H} \alpha / \mathrm{H} \beta$ ratio. We note that the SDSS fibers are on average smaller than the Ly $\alpha$ radii, but do capture the bulk of the nebular emission, and fluxes can easily be measured without contamination of $[\mathrm{NII}]$ and stellar absorption. Since Meurer et al. (1999) $\beta$ has been used almost ubiquitously as a proxy of stellar attenuation in high- $z$ galaxies; here we measure $\beta$ from aperture-matched HST imaging using the FUV (SBC/F140LP or F150LP) and the $U$-band (UVIS/F336W or F390W) filters. With colors between $\beta \approx-2.2$ and -0.6 our objects have similar UV slopes to the vast majority of those found in $z=2-4$ Ly $\alpha$-emitting galaxies (Blanc et al. 2011). Similarly the

\footnotetext{
${ }^{16} \mathrm{UV}$ continuum flux density, parameterized by a power-law of the form $f_{\lambda} \propto \lambda^{\beta}$.
}

$\mathrm{H} \alpha / \mathrm{H} \beta$ ratio is the canonical probe of nebular reddening (i.e. that which is to zeroth order expected for Ly $\alpha$ ) used in studies of low- $z$ and Galactic nebulae. $\beta$ and $\mathrm{H} \alpha / \mathrm{H} \beta$ are listed in Table 1 .

Both measures of dust content strongly anti-correlate with $\xi_{\mathrm{Ly} \alpha}$ although the sample is small ( $N=12$ defined sizes in $\operatorname{Ly} \alpha$ ). To assess its significance we compute the Spearman rank correlation coefficient, $\rho$, which yields $\rho=-0.73$ and -0.61 for the anti-correlation of $\xi_{\mathrm{Ly} \alpha}$ with $\beta$ and $\mathrm{H} \alpha / \mathrm{H} \beta$, respectively. This corresponds to likelihoods of the null hypothesis - that this correlation arises purely by chance - amounting to 0.7 percent (UV slope), and 3.6 percent $(\mathrm{H} \alpha / \mathrm{H} \beta)$.

The halo-dust phenomenon appears not to be a direct effect of radiative transfer. We have performed new test simulations with the McLya code (Verhamme et al. 2006), by tuning the gas-to-dust ratio in the synthetic galaxy of Verhamme et al. (2012). Indeed the surface brightness does scale with dust abundance but the light profile (therefore $R_{\mathrm{P} 20}^{\mathrm{Ly} \alpha}$ ) does not, and the $\xi_{\mathrm{Ly} \alpha}$-dust trend must be a secondary correlation. A scenario is needed in which galaxies decrease the relative size of their Hi envelopes as the absolute dust content increases. A sequence in which neutral gas settles into the galaxy (reducing $\xi_{\mathrm{Ly} \alpha}$ ) and subsequently forms stars (creating more dust) would explain the trend, but without yet having obtained spatially resolved Hi data this is conjecture.

Scattering also has the potential to spread Ly $\alpha$ over such an area that its surface brightness decreases greatly. In such a case, scattered radiation measured at large radii may not be sufficient to recover flux from a broad central absorption, making $\xi_{\mathrm{Ly} \alpha}$ observationally undefined when it is actually very large. The trend of $\xi_{\mathrm{Ly} \alpha}$ increasing in bluer galaxies, then, is also able to explain the undefined sizes of LARS 04 and 06, at their measured dust abundance. Similar considerations would also explain the non-detection of $\operatorname{Ly} \alpha$ in local gas-rich but metaland dust-poor dwarf starbursts such as IZw 18 and SBS 0335-052 (Kunth et al. 1994; Mas-Hesse et al. 2003; Ostlin et al. 2009), as discussed in Atek et al. (2009b).

We have empirically shown before (Atek et al. 2009a; Hayes et al. 2010) that the global escape fraction of Lya photons anti-correlates strongly with attenuation (also Kornei et al. 2010 in LBGs). We now demonstrate that 
at lower $E_{B-V}$, the more strongly emitting galaxies are likely to also spread their Ly $\alpha$ over larger surfaces. Thus while they do transmit more of their Ly $\alpha$, it may be that more of the transferred Ly $\alpha$ is observationally lost outside photometric apertures. This may also explain the lack of correlation between $\operatorname{Ly} \alpha / \mathrm{H} \beta$ and $E_{B-V}$ observed by Giavalisco et al. (1996), compared to trends seen in other samples: the aperture of the IUE probed just $3 \mathrm{kpc}$ at $z=0.01$ and if more Ly $\alpha$ is lost in bluer galaxies the Ly $\alpha /$ Balmer ratios would be artificially lowered in in such systems. This could in part mask an underlying correlation. By a similar token, galaxies that can very efficiently scatter Ly $\alpha$ photons may not be recovered at all, despite frequently showing very blue UV colors. Determining precisely how Ly $\alpha$ profiles are modified for a given set of host properties will provide a cornerstone for interpreting future large high- $z$ surveys.

M.H. received support from Agence Nationale de la recherche bearing the reference ANR-09-BLAN-0234-01. G.Ö. is a Swedish Royal Academy of Sciences research fellow supported by a grant from Knut and Alice Wallenberg foundation, and also acknowledges support from the Swedish research council (VR) and the Swedish National Space Board (SNSB). A.V. benefits from the fellowship 'Boursière d'excellence de l'Université de Genève'. H.A. and D.K. are supported by the Centre National d'Études Spatiales (CNES) and the Programme National de Cosmologie et Galaxies (PNCG). I.O. acknowledges the Sciex fellowship. H.O.F. acknowledges financial support from CONACYT grant 129204, and Spanish FPI grant BES-2006-13489. H.O.F. and J.M.M.H. are partially funded by Spanish MICINN grants CSD200600070 (CONSOLIDER GTC), AYA2010-21887-C04- 02 (ESTALLIDOS) and AYA2011-24780/ESP. We thank C. Steidel for making the high- $z$ Ly $\alpha$ profiles available for our comparisons in Section 4 .

Facilities: HST (ACS,WFC3).

\section{REFERENCES}

Adams, J. J., Blanc, G. A., Hill, G. J., et al. 2011, ApJS, 192, 5

Atek, H., Kunth, D., Hayes, M., Östlin, G., \& Mas-Hesse, J. M. 2008, A\&A, 488, 491

Atek, H., Kunth, D., Schaerer, D., et al. 2009a, A\&A, 506, L1
Atek, H., Schaerer, D., \& Kunth, D. 2009b, A\&A, 502, 791

Barnes, L. A., \& Haehnelt, M. G. 2010, MNRAS, 403, 870

Blanc, G. A., Adams, J. J., Gebhardt, K., et al. 2011, ApJ, 736, 31

Calzetti, D., Armus, L., Bohlin, R. C., et al. 2000, ApJ, 533, 682

Cannon, J. M., Skillman, E. D., Kunth, D., et al. 2004, ApJ, 608, 768

Cowie, L. L., \& Hu, E. M. 1998, AJ, 115, 1319

Feldmeier, J., Hagen, A., Ciardullo, R., et al. 2013, ArXiv e-prints Fynbo, J. P. U., Ledoux, C., Möller, P., Thomsen, B., \& Burud, I. 2003, A\&A, 407, 147

Fynbo, J. U., Möller, P., \& Thomsen, B. 2001, A\&A, 374, 443

Giavalisco, M., Koratkar, A., \& Calzetti, D. 1996, ApJ, 466, 831 Guaita, L., Gawiser, E., Padilla, N., et al. 2010, ApJ, 714, 255

Hayes, M., Östlin, G., Atek, H., et al. 2007, MNRAS, 382, 1465

Hayes, M., Östlin, G., Mas-Hesse, J. M., \& Kunth, D. 2009, AJ, 138,911

Hayes, M., Östlin, G., Mas-Hesse, J. M., et al. 2005, A\&A, 438, 71

Hayes, M., Östlin, G., Schaerer, D., et al. 2010, Nature, 464, 562

Kornei, K. A., Shapley, A. E., Erb, D. K., et al. 2010, ApJ, 711, 693

Kunth, D., Lequeux, J., Sargent, W. L. W., \& Viallefond, F. 1994, A\&A, 282, 709

Laursen, P., Razoumov, A. O., \& Sommer-Larsen, J. 2009, ApJ, 696,853

Mas-Hesse, J. M., Kunth, D., Tenorio-Tagle, G., et al. 2003, ApJ, 598,858

Matsuda, Y., Yamada, T., Hayashino, T., et al. 2012, MNRAS, 425,878

Meurer, G. R., Carignan, C., Beaulieu, S. F., \& Freeman, K. C. 1996, AJ, 111, 1551

Meurer, G. R., Heckman, T. M., \& Calzetti, D. 1999, ApJ, 521, 64

Mosleh, M., Williams, R. J., Franx, M., \& Kriek, M. 2011, ApJ, 727,5

Neufeld, D. A. 1990, ApJ, 350, 216

Nilsson, K. K., Tapken, C., Møller, P., et al. 2009, A\&A, 498, 13

Östlin, G., Hayes, M., Kunth, D., et al. 2009, AJ, 138, 923

Ouchi, M., Shimasaku, K., Akiyama, M., et al. 2008, ApJS, 176, 301

Petrosian, V. 1976, ApJ, 209, L1

Prescott, M. K. M., Dey, A., \& Jannuzi, B. T. 2012, ApJ, 748, 125

Rauch, M., Haehnelt, M., Bunker, A., et al. 2008, ApJ, 681, 856

Rhoads, J. E., Malhotra, S., Dey, A., et al. 2000, ApJ, 545, L85

Steidel, C. C., Bogosavljević, M., Shapley, A. E., et al. 2011, ApJ, 736,160

Verhamme, A., Dubois, Y., Blaizot, J., et al. 2012, A\&A, 546, A111

Verhamme, A., Schaerer, D., \& Maselli, A. 2006, A\&A, 460, 397

Yun, M. S., Ho, P. T. P., \& Lo, K. Y. 1994, Nature, 372, 530

Zheng, Z., Cen, R., Weinberg, D., Trac, H., \& Miralda-Escudé, J. 2011, ApJ, 739, 62 